\title{
Here is the author! Hyperlinks as constitutive rules of hypertextual communication
}

SABRINA MAZZALI-LURATI

\section{Abstract}

In this paper, we examine the importance of hyperlinks in revealing the presence of the hypertext author and his decisive role in defining and controlling the dialogue with the user/reader through the hypertext. This is essential in order to adequately describe all the factors involved in hypertextual communication and how this communication takes place. In hypertexts and hypermedia, hyperlinks on the one hand define the possible directions of hypertextual communication and, on the other hand, their strategy of manifestation shapes the user's process of interpretation. Because of these two important 'powers' hyperlinks have, and on the basis of John Searle's distinction between constitutive and regulative rules, hyperlinks can be defined as constitutive rules of hypertextual communication, set by the author, needing to be activated by the reader, and determining different utterances in the interaction happening during the navigation between the author and the user/reader. In the paper, after having described the semiotic-communicative structure of hyperlinks and the process of interpretation they require, the character of constitutive rules of hyperlinks will be illustrated by analyzing different dialogues generated by different hyperlinks in two particular kinds of hypermedial applications: hypertextual transpositions of classic literary texts and hyperfiction.

Keywords: semiotics; hypermedia; hyperlinks; constitutive rules; dialogue; author.

\section{Introduction}

Hyperlinks are a central element of hypertext. As most of the well-known definitions show (cf. Nelson 1990; Slatin 1990: 877; Bolter 2001: 35; 
Bolter and Grusin 1999: 272; cf. also Cantoni and Paolini 2001: 36), the presence of hyperlinks is part of the definition of hypertext itself. It is commonly acknowledged that hypertext is

the use of the computer to transcend the linear and fixed qualities of the linear text. Unlike the static form of the book, a hypertext can be composed, and read, non-sequentially; it is a variable structure, composed of blocks of text (or what Roland Barthes terms lexia) and the electronic link that join them. (Delany and Landow 1994: 3)

Similar devices can be found in other kinds of printed communication artefacts (we refer mainly to books). Superscript numbers related to footnotes or endnotes, cross-references, and entries of analytic indices offer the reader the possibility of exploring non-linear reading paths. However, the presence of these devices is not essential to the artefacts themselves. Not all books have analytic indices and the presence of cross-references in the text and of footnotes or endnotes with superscript numbers related to them is not a definitional element of a book. Moreover, the role these devices play in text consumption is much more limited. The act of reading can happen independently of the activation of these devices, while the consumption of hypertext relies on the activation of hyperlinks. The reader's consumption cannot advance without the mechanical activation of one of the hyperlinks available on the page.

It is commonly maintained (cf. for example Joyce 1995; Landow 1997) that the presence of hyperlinks in hypertext introduces a new challenge to the act of reading and interpreting. Hyperlinks are responsible for multilinearity, the main characteristic of hypertext, capable of modifying the relationship between author and reader. Thanks to multilinearity, the hypertext reader is not constrained by a predefined reading order; he can choose the reading path he prefers. It is up to him to decide what to read and in which order. Because of this freedom, the hypertext reader appears to occupy a leading position with respect to the author. The construction of meaning seems to depend mainly on his choices. The presence of hyperlinks seems to accord prominence to the reader and to overshadow the author.

In this paper, we examine the importance of hyperlinks in revealing the presence of the hypertext author and the decisive role the author plays in defining and controlling the dialogue with the reader through hypertext. Our hypothesis is that hyperlinks are constitutive rules of hypertextual communication set by the author and needing to be activated by the reader. These constitutive rules determine different utterances in 
the interaction happening through the application. This character of constitutive rules of hyperlinks can be described and we consider that, by casting light on it, it is possible to adequately consider all the factors involved in hypertextual communication. Namely, it is possible to attach the proper importance to the role of the author. Through hyperlinks (by setting the constitutive rules), the author remains present in the hypertextual dialogue more than he can in other kinds of asynchronous communication.

Our description of what characterizes hyperlinks as constitutive rules of hypertextual communication will be based on some examples of hyperlinks available in two different but connected kinds of hypermedial applications: hypertextual transpositions and hyperfiction. Hypertextual transpositions are off-line or online hypermedial applications devoted to the presentation of classic literary texts, consisting of the electronic version of the literary text and of other texts, images, video clips, and audio files that help in understanding the literary text itself and in enriching the reading experience. Hyperfiction is a new literary genre (the knowledge of which has until now remained confined to a restricted community), initiated at the end of the 1980s and aimed at exploiting the new possibilities offered by the hypertextual form for the creation of literary works. The interest in these categories of applications is that, although they belong to the same field (literature), they use different kinds of textual procedures and, correspondingly, they establish different kinds of dialogues with the reader. Hypertextual transpositions are mainly explanatory and expositive texts, while hyperfictions are mainly narrative texts. Through hypertextual transpositions, the dialogue is about the classic literary text (with the goal of sustaining the reader's comprehension of its significance), while in hyperfiction the narration itself is the goal of the dialogue. Hyperfiction is also interesting because hypermedia belonging to this category experiment with the use of multilinearity to allow the reader the widest possible margin of freedom in meaning construction to the point of disorientation, which is held to be a 'positive' rhetorical device ('... hyperfiction writers seem to look for disorientation as a creative value' [Pajares Tosca 1999: 217]).

In describing what characterizes the constitutive rules of hyperlinks, we will use examples drawn from two hypertextual transpositions of Shakespeare's A Midsummer Night's Dream (lingo.uib 1999; BBC Education 1996, referred to hereafter as MD1 and MD2, respectively), three hypertextual transpositions of Shakespeare's Macbeth (BBC Education 1995; Voyager 1994; Bride Digital Classics 1999, referred to hereafter as M1, M2, and M3, respectively) and the hyperfiction Lasting Image by Carolyn Guyer and Michael Joyce (2000). 


\section{The semiotic-communicative structure of hyperlinks}

What characterizes hyperlinks as constitutive rules of hypertextual communication depends on their semiotic-communicative structure.

Because of their well-acknowledged crucial importance in hypertext, hyperlinks have been widely studied from different perspectives in order to describe their intrinsic nature. Studies of hyperlinks from a pragmatic perspective have cast light on essential aspects of hyperlink structure. Cantoni and Paolini (2001: 42) define hyperlinks as 'actions being performed depending on reader choices' and corresponding to dialogical utterances of the kind '[I want] something [to happen],' which are sometimes quite similar to performative sentences. Bernd Wingert (1999) emphasizes that every link creates in the user an expectation that the target has to fulfill. In the same line, starting from Sperber and Wilson's relevance theory, Susan Pajares Tosca (2000) outlined a specific presupposition of hypertext and of hyperlink, suggesting that

... links communicate a presumption of [their] own optimal relevance. That is, if a word (or picture) is highlighted, the reader has to understand that it points to a relevant development of the text. Links don't interrupt the flow of meaning; on the contrary, they enliven it. (Pajares Tosca 2000: 80)

Such a promise of relevance is an essential component of hyperlink meaning, to which another component has to be added: the characteristic of invitation. The link meaning comprises two aspects. First, the link is a proposal, an invitation from the author for the continuation of communication. It is an indication (provided by the author to the reader) of possible further directions of communication. Second, this proposal contains a promise of relevance. In other words, every link presents a semanticpragmatic function. The pragmatic aspect corresponds to the invitation addressed by the author to the reader and including a promise of relevance with respect to the reader. The semantic aspect consists in the (more or less strong) semantic relationships existing between the source node and the target node and in the interest this semantic relationship has to the reader.

As with every sign, besides the intelligible part consisting in their function (meaning), hyperlinks present a perceptible part, a strategy of manifestation (the anchor). Similar to its meaning, the hyperlink strategy of manifestation consists of two elements. First, it consists of a perceptible graphical change in the content displayed on the screen. It can be an underlining, a different color in the writing, a different color in the 
background, a button-shape border, a change of the cursor's shape, etc., and it signals the presence of a physical spatial path from the source to the target. In this respect, and according to Peirce's classification of signs, the anchor is an indexical sign: it indicates the presence of a connection between source and target, the presence of an author's invitation (cf. also Wenz 1997; Fagerjord 2001; Wirth 2002). Second, an anchor also always entails another element: a word, a linguistic expression, a number, a diacritic sign, a drawing, an image, etc. Besides visual elements manifesting the indexical aspect, the anchor includes another sign that can in itself be an index, an icon, or a symbol. For example, a word within a text in the node can be the anchor for a link. This anchor is constituted not only of the underlining, but also of the symbolic sign itself (i.e., the word). Therefore, as a whole, the anchor of a link is an indexical, an iconic, or a symbolic sign to which some indexical visual elements are attached in order to transform the indexical, iconic, or symbolic sign into an indication to the user of the existence of a possible continuation of the navigation. The task of the incorporated indexical, iconic, or symbolic sign is to manifest the semantic relationship between source and target by the manifestation of the semantic relationship between the anchor and the target.

Hyperlinks are complex signs presenting a two-fold structure both at the level of their strategy of manifestation and at the level of meaning. Their communicative structure also contributes to their relationship to the addresser and to the addressee. In Karl Bühler's terms (1999 [1934]), we can say that hyperlinks have a function of expression and a function of appeal. On the one hand, behind every link there is an author who decided to establish it and who decided which anchor was the most adequate. On the other hand, every link will be perceived differently by different readers (otherwise, how to explain that a reader chooses a link and that another reader chooses another link?).

\section{The process of hyperlink interpretation}

When clicking on a link, the reader/user has to first understand what the relationship is between the anchor (the perceptible part of a link) and the target and second what the relationship is between the node of departure ('source') and the node of arrival ('target'). Because of the semioticcommunicative structure hyperlinks have, the process of interpretation they require is complex. It derives from the combination of different inferences allowing an understanding of the different elements composing the hyperlink. 
From a pragmatic point of view, on the basis of Sperber and Wilson relevance theory, Susan Pajares Tosca $(1999,2000)$ underlines the 'lyrical quality of hypertext.' 'Lyrical' has to be understood in the sense described by Pilkington (2000), meaning 'a particular kind of interpretive strategy' characterized by a 'particular intensity in the search for meaning, similar to the way we read poetry' (Pajares Tosca 2000: 83). This 'lyrical quality' stems from the fact that hyperlinks interpretation happens through a double inference. The first inference takes place at the moment of the choice of the link the reader wants to click on and it consists of the search for plausible implications related to the anchor. The second inference consists in the adjustment of the implications generated in the first inference with the actual contents of the target node. Even if it describes the reader's reasoning as being much more systematic than it actually is in common web navigation sessions (it is arguable that when choosing a link a reader specifies all the possible inferences and that the relationship between the first and the second level of inference is as logical as it appears in such a framework), such an approach is interesting, because it identifies the central element of the complexity of hyperlink interpretation, from which stem the problems of disorientation and cognitive overhead that have been discussed in relation to hypertext consumption since its inception (cf. Conklin 1987).

By considering this central element of the complexity of hyperlink interpretation from a semiotic-hermeneutic point of view, we observe that the reader always applies two different (interlaced) processes of interpretation. ${ }^{1}$ On the one hand, in order to understand the aspect of the author's invitation through a link's meaning (that is, in order to understand the indexical aspect of the anchor), the reader applies a symptomatic (indexical) process of interpretation. A symptomatic process of interpretation is a process in which the understanding of the relationship existing between the anchor and the hyperlink function is based on causality. The reader's reasoning can be paraphrased as follows: 'since at this point of the hypertext there is an anchor, there also is an author's proposal for the continuation of the communication.' On the other hand, the aspect of the link's meaning corresponding to the semantic relation (which is an important element of link's relevance) can be interpreted through an iconic or a symbolic process of interpretation. An iconic process of interpretation is a process in which the understanding of the relationship existing between anchor and hyperlink function is based on an association. A symbolic process of interpretation is a process in which the understanding of the relationship existing between anchor and hyperlink function is based on the application of rules. A hyperlink is interpreted through an iconic process when the sign included in the anchor lets the user infer the 
target and its relevance with respect to the source thanks to the recognition of the associative relationship it maintains to the target. A hyperlink is interpreted through a symbolic process when the sign included in the anchor lets the user infer the target and its relevance with respect to the source thanks to the user's knowledge of some rules valid in the application. It can also happen that the relevance cannot be inferred, because the indexical, iconic, or symbolic sign incorporated in the anchor is not adequate. In such cases, what remains is just the first aspect, the indexical one. The user clicks on such a link only because he trusts the author who, setting this particular link, states that a continuation of the navigation in this direction is possible and worthwhile. He just employs a symptomatic process of interpretation. What is interesting is that the promise of relevance is assumed in any case. Despite the fact that the indexical, iconic, or symbolic sign included in the anchor is opaque, this presupposition does not fall. On the contrary, it is because of the existence of this presupposition that the user can trust the author despite the opacity of the anchor. ${ }^{2}$ It has to be noted that, as the navigation goes on and the reader learns the regularities of the application and its language, all processes of hyperlink interpretation tend to become symbolic.

The ' $M$ ' clapperboard link in figure 1 is interpreted through an iconic process of interpretation. The anchor is composed of an iconic sign, a drawing of a clapperboard, on which the initials of the name of the tragedy's main character (which is also the title of the tragedy) appear. Through an association, the user infers that, clicking on that link, he accesses a video clip representing a sequence of the play. Subsequently (once the user has verified the correctness of such inference and, thus, learned a rule), the process of the interpretation of this hyperlink becomes symbolic. The page number hyperlink is interpreted through a symptomatic process of interpretation. This link provides access to the characters collection center (from which the user can access description cards of all characters). It is a 'hidden' link; no sign allowing the user to recognize it as a link is present. Elements that usually manifest the indexical aspect of the link are absent. The anchor is composed of a number, which is an index with respect to the displayed text's page. No semantic relationship exists between the anchor and the target. The user cannot foresee what he will find by clicking on it. He clicks only by trusting the promise of relevance and by recognizing the existence of a possibility of continuation of the navigation. After having clicked on this link, he learns the application rule according to which by clicking on this link it is possible to access to the collection of the characters' descriptions. From this moment on, the process of interpretation becomes symbolic. 

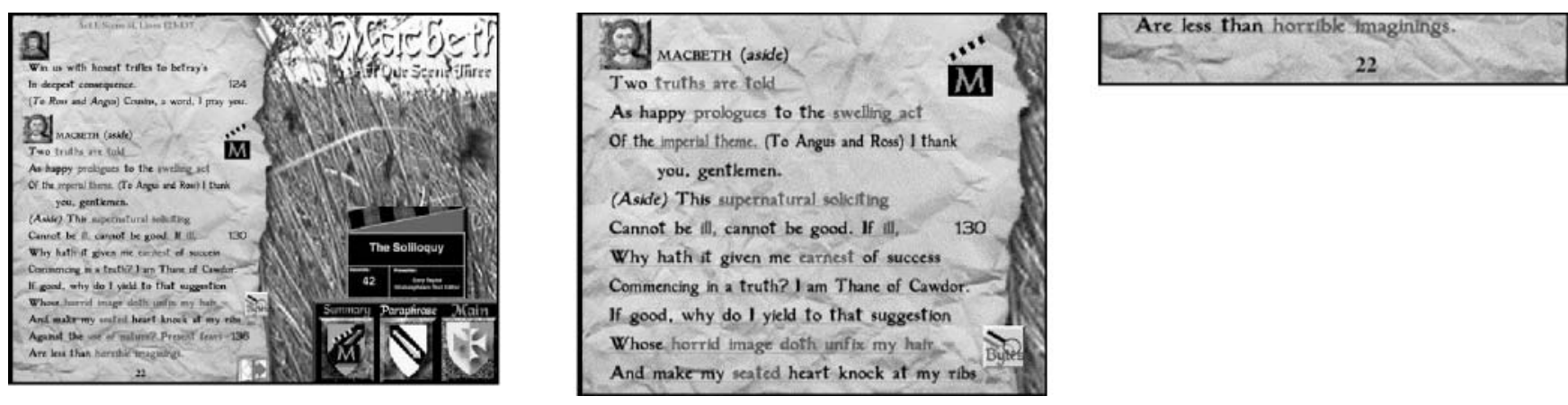

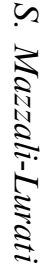

Figure 1. Text of the play screen (left) and details of some of the available hyperlinks (center and right) from M3 


\section{Hyperlinks as constitutive rules}

We develop the issue of rules referring to John Searle's distinction between regulative and constitutive rules (1995 [1969]: 33-42). Usually, we conceive of the existence of rules applied to behaviors, attitudes, or activities that exist independently of the rules themselves. This is the realm of regulative rules, which function as orders (for instance 'In order to reach Milan from Lugano, drive through Chiasso'). However, Searle pointed out the existence of another kind of rule, which cannot be separated from the behavior, attitude, or action. It is, for example, the case of the rules concerning the moves of the queen in chess. Were these rules suspended or not yet established, the connected behaviors and actions (corresponding to the chess play) would not exist. The activity of pushing a piece of wood on a table of eight times eight squares would, but this would not be chess playing. Searle defined these kinds of rules as constitutive, meaning that the connected behavior, attitude, or activity does not exist without the rule itself.

At first sight, hyperlinks seem to belong to the category of regulative rules. Starting from the common view of hypertext as a paradigm of possible choices enhancing the user's freedom in the content of consumption (which is a basic principle and the very novelty of hypertextual communication), hyperlinks can be described as rules of the kind 'To have information about [a given topic or issue] click on this link' or 'In order to perform this operation, click this link' (which are very similar to an order of the kind 'In order to reach Milan from Lugano, drive through Chiasso'). Such an interpretation of hyperlinks focuses only on one aspect of hypertextual communication where attention is geared towards a conception of the application as a database, a repository, where the user can choose what he wants or needs.

However, there are aspects of hyperlinks that make them more similar to constitutive rules. Hypertextual communication cannot exist independently from the hyperlinks proposed by the author and chosen by the reader. Besides, the proposition and the choice of different hyperlinks constitute different dialogues. As a consequence, the most adequate paraphrase of hyperlinks meaning is of the kind 'This hyperlink counts as a given (kind) of utterance in the context of this reading path' (cf. Pajares Tosca 2000: 79, who states that hypertext nodes and its links can be considered speaker's utterances). In this respect, hyperlinks appear to be constitutive rules of hypertextual communication. This characteristic of constitutive rules stems from the power hyperlinks have in conditioning and defining two basic processes involved in hypertextual communication, namely the navigation and the user's interpretation. In other words, 
hyperlinks can be considered to be constitutive rules of hypertextual communication because, first, they define the possible directions in the communication and, second, they define the user's process of interpretation (their form, their strategy of manifestation, conditions the user's interpretation in a decisive manner). In the following, we will describe how this happens and how this gives birth to different dialogues.

\subsection{Hyperlinks define the possible directions of the communication}

The power hyperlinks have in defining and conditioning the progression of the communicative exchange is quite evident. Notwithstanding the enthusiasm for the possibility of realizing the reader's freedom that vividly appeared at the beginning of hypertext's flourishing (cf. for instance Landow 1997), the practice of hypermedia design and the studies on hypermedia consumption, web usability, and requirement analysis soon proved that such a freedom can only be limited to the range of possibilities offered by the author/designer.

However, it is interesting to describe some examples showing the consequences of the power of hyperlinks to condition the progression of the communication, because interesting implications as to the kind of generated dialogues will emerge.

A hyperlink is always established by the author. It is up to the author to decide if and which hyperlinks have to be made available at a given point of the hypertext. ${ }^{3}$ In this way the author decides that the communication can go on in the directions $a, b$, or $c$ or in the directions $d, e$, or $f$. Depending on these choices by the author, a different communicative act will be generated through the reader's navigation.

As an example we can consider the different choices made by the authors of MD1 and MD2. On the text pages of MD2, hyperlinks leading to explications of given passages of the text are available. Such explanations are provided in pop-up windows that are displayed simultaneously with the text of the play. In the pop-up windows, no links connecting to other explications are present. Owing to this author's choice the reader's attention is kept on Shakespeare's text and the reader is always brought back to the act of reading the text of the play (cf. figure 2).

The author of MD1 provides hyperlinks not only between the text of the play and the explications, but also between the different explications (of different passages of the text of the play). Because of this author's choice the reader can leave the act of reading the text of the play and begin to follow different explicative thematic paths (cf. figure 3). 


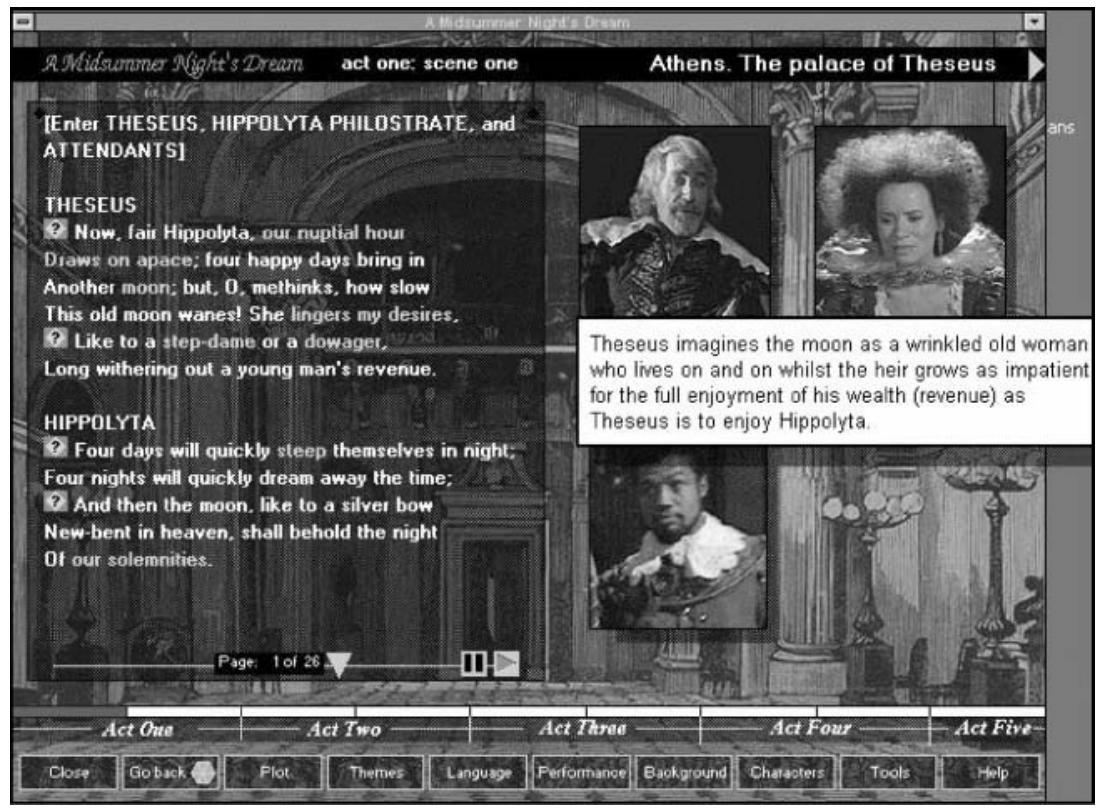

Figure 2. In MD2, explanations of Shakespeare's text are provided in pop-up windows. No links allowing the visualization of another explanation are available in the pop-up windows

Starting from Grice's principle of cooperation (1995 [1975]: 204), we can say that the hypertext author decides to provide certain hyperlinks instead of others at a given point of the hypertext because he considers such hyperlinks to be more appropriate than others from a conversational point of view. In other words, the author considers such hyperlinks to correspond to the goal and the orientation of the exchange he aims at establishing with the reader and for which he composed the hypertext. For example, the author of MD2 considered it more appropriate for the reader to read Shakespeare's text, while the author of MD1 considered more important to the reader the possibility of deepening the knowledge of the play through the exploration of different thematic paths. The choice of the author of MD2 can be paraphrased as an utterance of the kind 'Here is a useful explanation to the text you are reading,' while the choice of the author of MD1 corresponds to an utterance such as 'You can find also other interesting and related explanations.' The constitutive rule corresponding to the hyperlink of MD2 can be paraphrased as 'This hyperlink counts as a brief explanatory digression from the reading of the text of the play.' The constitutive rule corresponding to 


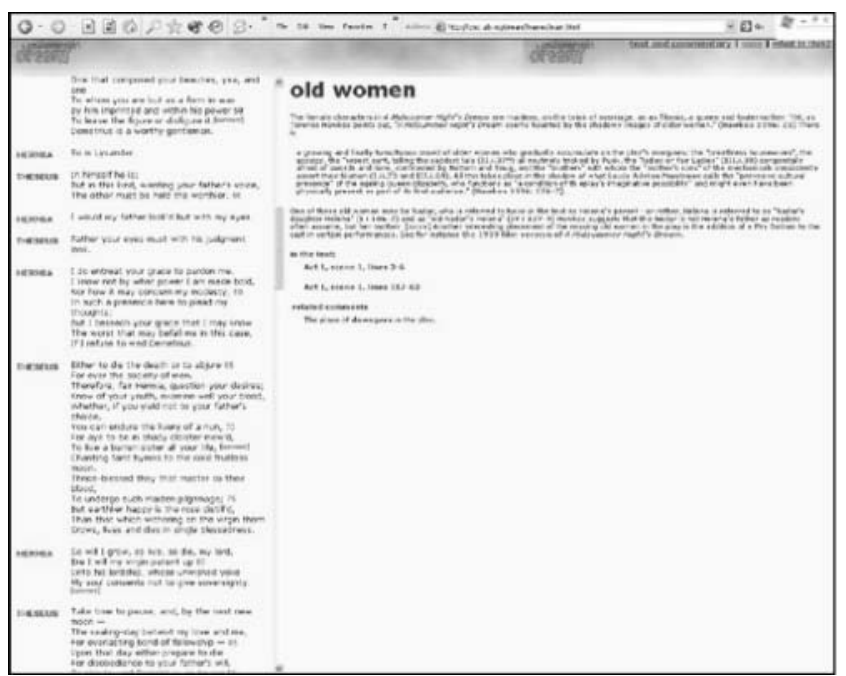

\section{old women}

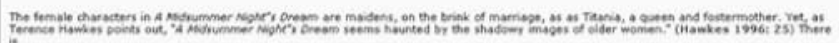

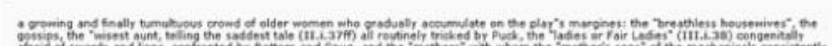

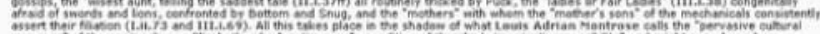

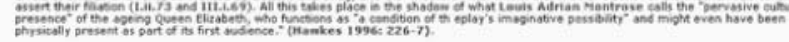

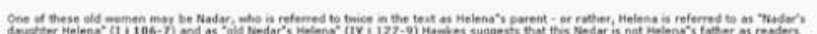

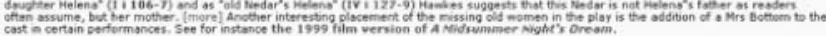
in the terit:

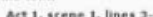

Aet 1, seene L, lines isz 60

The place of domagers in the plar.

Figure 3. Annotations in MD1 that the reader can access to from the literary text's screens contain several embedded links, leading to other (thematically or logically related) annotations 
the hyperlink of MD1 can be paraphrased as 'This hyperlink counts as a proposition of thematically related commentaries.'

Similarly, in M3 an arrow hyperlink leading to the following page of the text of the play is available on all text pages. However, on the last page of a given act this hyperlink does not bring the reader directly to the first page of the following act, but it leads the reader on the main menu. This suggests that the author of M3 perceives (and tries to lead the reader to the same perception) that the reading of the pages belonging to an act is a unity which has not to be interrupted, while at the end of a given act it can be appropriate to jump to another act instead of continuing the act of reading in a linear way (cf. figure 4).

On the contrary, in M1 and M2 a hyperlink allowing the continuation of sequential reading between different acts is also present (cf. figures 5 and 6). The constitutive rule corresponding to these hyperlinks can be paraphrased as 'This hyperlinks counts as a statement (to which the reader is presupposed or obliged to agree) about the suitability of continuing the reading in following the linear order of Shakespeare's text.' The constitutive rule corresponding to the forward arrow hyperlink available in M3 on the last page of each act can be paraphrased as 'This hyperlink counts as a signalling that the end of a unity was reached and that, consequently, at that point, the reading order can be changed.'

Since the author considers some hyperlinks as being more appropriate, from a conversational point of view, in respect to the goal he aims at sharing with the reader, it is very likely that he will use technical (particularly, graphical) devices in order to highlight them and direct the reader's attention on them. For instance, in Lasting Image two kinds of hyperlinks are available on each page (cf. figure 7). Hyperlinks carrying to a logical and immediately coherent reading of the story (consisting in the following sequence: presentation of the time and place of the narrated events and introduction of the narrating voice; introduction of the main character; story of the main character; narration of the life of the narrating voice; narration of a fact connecting the life of the narrating voice and the main character) are visible at the bottom of each page. Their anchor consists in backward/forward arrows and their visibility immediately strikes the reader. By following them the reader can easily reconstruct the narration. He is brought to a reading in which the immersion (deriving 'from our being completely absorbed within the ebb and flow of a familiar narrative schema' [Douglas and Hargadon 2000: 154]) is prominent. On the contrary, hyperlinks leading to a reading of the story requiring more interpretive work for the reader (owing to the presence of jumps in logic, analepses and prolepses, poetic remarks on events or places of 


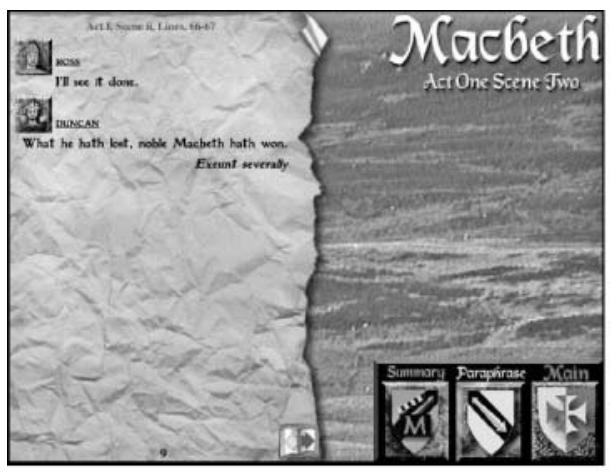

\section{Jacbeth}

\section{Act One Scene Four}

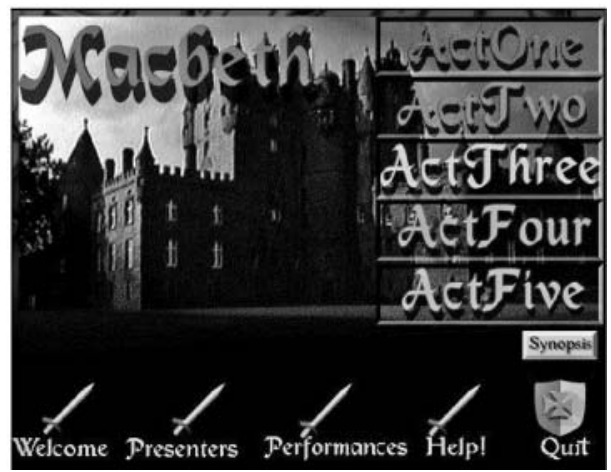

Figure 4. In M3, the forward arrow hyperlink available on the last page of a given scene leads directly to the beginning of the following scene (top left and right), while the forward arrow hyperlink available on the last page of a given act leads on the main menu (bottom left and right) 

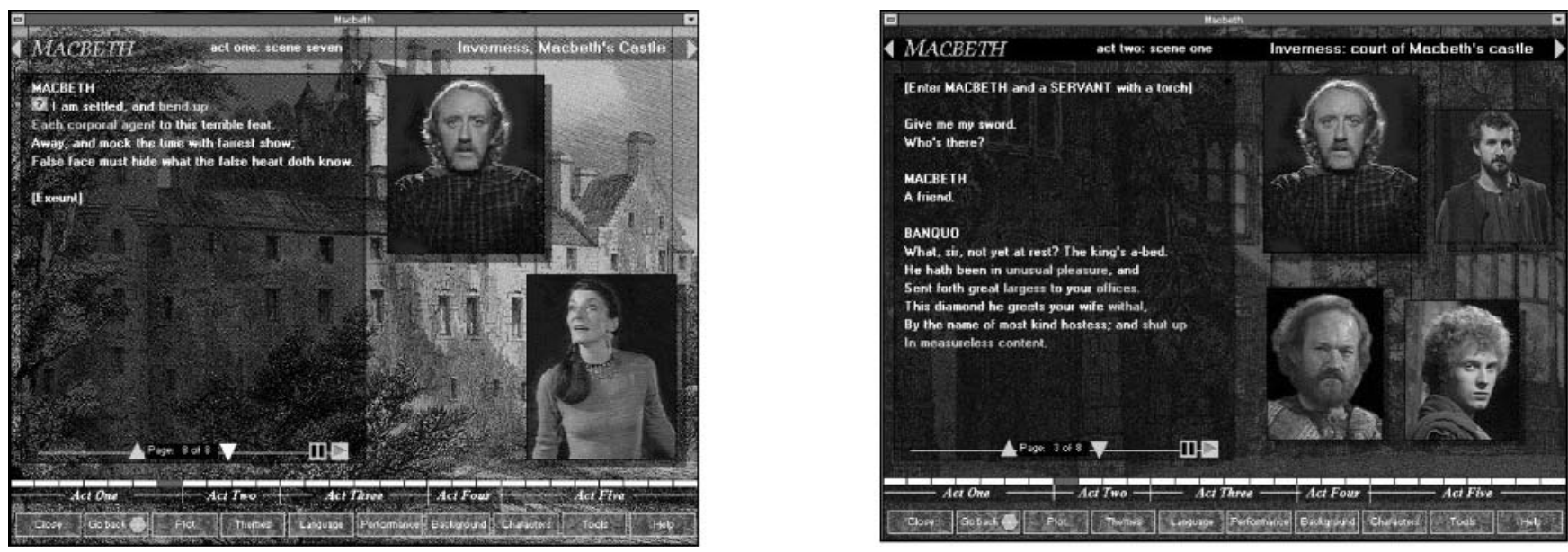

Figure 5. In M1, the forward arrow hyperlink available on the last page of a given act (left) leads directly to the first page of the following act (right) 

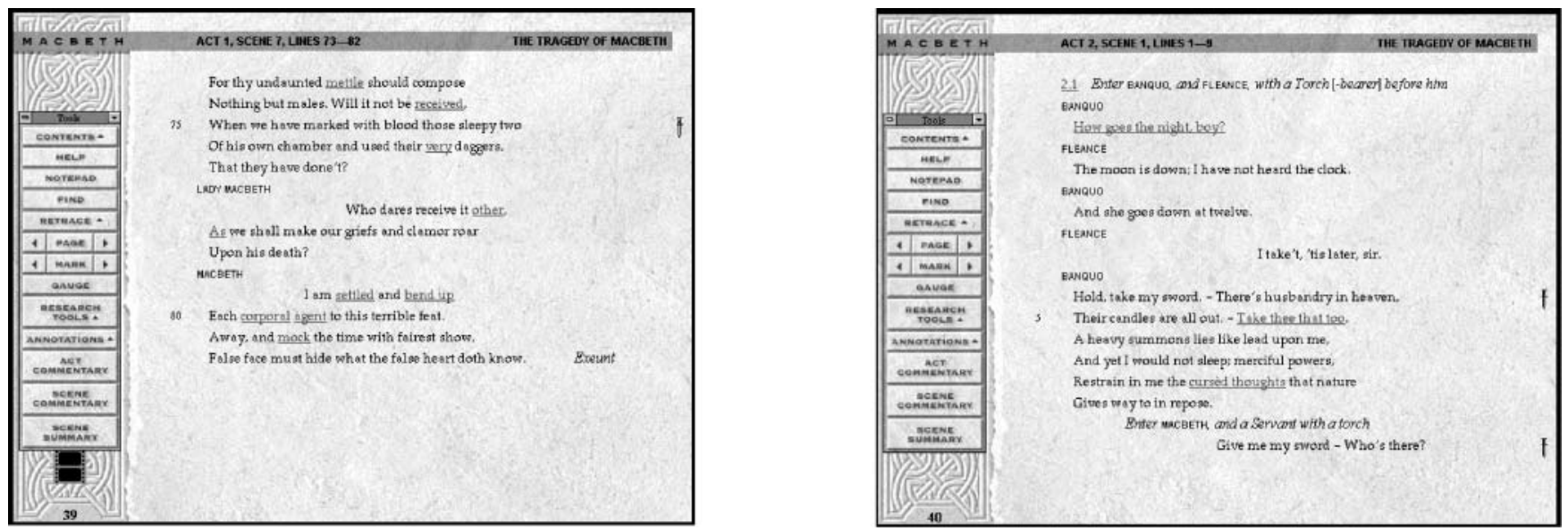

N

$\stackrel{T}{N}$

Figure 6. In M2, the forward arrow hyperlink available on the last page of a given act (left) leads directly to the first page of the following act (right) 

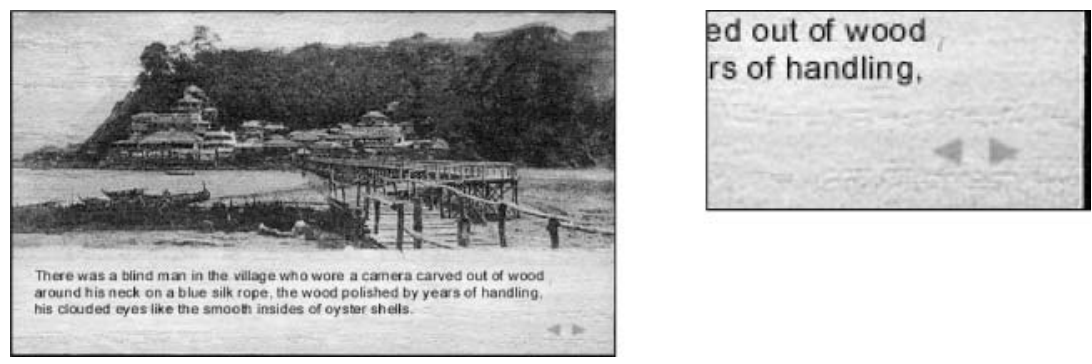

Figure 7. In Lasting Image, on each text page forward and backward arrow hyperlinks are available. Furthermore, parts of the image and some words of the text of each page constitute the anchor of other (invisible) hyperlinks

the story) are invisible. Their anchors consist of parts of the text or the image. The reader has to look for them by moving the mouse on the page until the cursor shape changes from an arrow to a pointing finger. This is an intriguing activity that suggests an intriguing reading, in which the reader is led to interpret given elements or parts of the story in a way that he is obliged to change afterwards in order to save the coherence of the narration. These hyperlinks invite the reader to a reading in which engagement (consisting in the recognition of the absence of a usual and unique schema and of overturns and conflicts in the narration [Douglas and Hargadon 2000: 154]) is central.

Thanks to their visibility, the arrow hyperlinks are prominent for a novice reader. At first sight, they appear to be the only available links. In this way, the author directs the novice reader to the simplest reading. As long as the navigation goes on and the reader becomes familiar with hyperfiction, he discovers the hidden hyperlinks. The author invites such an expert reader to different possible reading paths capable of challenging his interpretive skills.

\subsection{Hyperlinks define the reader's process of interpretation}

The use of a given device instead of another in the strategy of manifestation conditions and defines the reader's process of interpretation of the hyperlink. Starting from Pajares Tosca's framework of hyperlink interpretation, we observe that a different strategy of manifestation gives rise to a different exploration of the possible meanings of the link both with the source node and with the target node. The different inferences provoked in the reader by different strategies of manifestation enrich and complete the sense gained by the reader in a different way. In particular, 
the strategy of manifestation affects the first level of the double inference process provoked by hyperlinks. In fact, at this first level of inference, the strategy of manifestation can provide hints about the content of the target node (and in this case the second level of inference will consist in confirming or specifying the implicatures generated at the first level); it can give rise to implicatures that will be completely revised at the second level of inference (this happens quite often in hyperfiction); it can provide no hints about the content of the target node (in this case the first level of inference consists uniquely in the implicature derived by the promise of relevance entailed by every link).

As an example, we can consider the links providing access to linguistic and cultural explanations of expressions of the Shakespearean text used in M1, M2, and M3. These links are 'hypertextual relatives' of superscript numbers used for footnotes or endnotes in printed editions and they realize the practice of literary annotations.

On the text pages of M1 two kinds of link are available: the question mark links and embedded textual links (cf. figure 8). Both kinds of link provide access to linguistic and/or cultural explanations clarifying the words or expressions of the text of the play and/or recalling elements of the plot that have to be kept in mind in order to understand the text. The difference between the two kinds of link consists in the fact that the explanations provided through the embedded textual links relate to the specific words composing the anchor, while the explanations provided through the question mark links relate to a wider passage of the text, side to which the link is set. This difference gives rise to a different implicature at the first inference level. The range of possible implicatures arising in front of the question mark links is wider than the range of implicatures arising in front of the embedded textual links. In relation to the question mark links, besides the incertitude concerning the precise kind of provided explanatory information - linguistic definition, translation into modern English, explanation of cultural and historical aspects allowing comprehension, reminder of previous facts or aspects of the plot (an incertitude that is common in the practice of literary annotation) - there is an incertitude as to the precise passage of the text of the play that is meant to be explained. From a semiotic-hermeneutic point of view, both links are interpreted through an iconic process of interpretation. However, when interpreting the question mark link, the association is expected to exist between the passage of the text to which the link refers and the kind of content of the target node (which is expected to be an answer about a question on the meaning of a not very well-delimited portion of text). When interpreting the embedded textual link, the association is expected to exist between the meaning of the words composing the anchor 

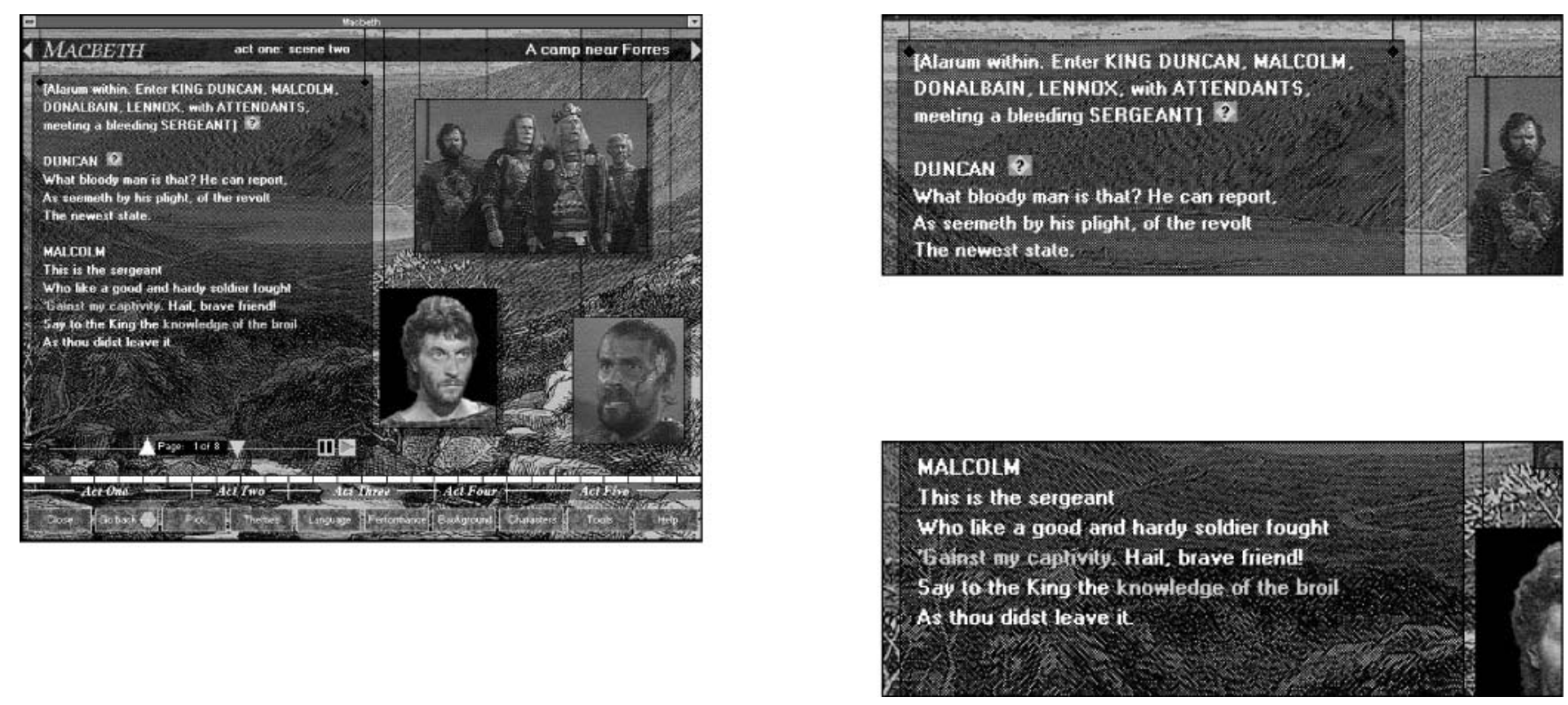

Figure 8. In M1, question marks and textual embedded hyperlinks are available on each text page 
and the content of the target node (the reader expects almost equivalence, synonymy between source, anchor, and target). In the perspective of a dialogue, the question mark link (and its target node) correspond to a quite complex author's utterance of the kind 'This hyperlink counts as a relevant explanation of the passage of the text side to which the link is set and the boundaries of which will be clear after having read the explanation itself; the content of the explanation belongs to the range of usual contents of literary annotations.' The verbal embedded link corresponds to an utterance of the kind 'This hyperlink counts as an explanation of the meaning of these words.'

A similar situation can be found in M2. On the text pages, embedded textual links provide access to linguistic explanations of the words composing the anchor (cf. figure 9). Their function is identical to the textually embedded links in M1. Besides, other links leading to linguistic explanations of wider passages of the text are available. These links are very similar to the question mark links of M1. They provide access to the same kind of content and they refer to a similar element (a wide passage of the text of the play). However, because of their different anchors, these two kinds of link provoke a different kind of inference. The question mark anchors in M1 bring the reader to implicatures of the kind 'In relationship to this passage there is an interesting question to be answered.' The shape of the link helps in signalling that the content of the target page is something that can clarify the passage of the text. This helps the reader in inferring that the content will be an explanation of some type. The anchor of the links in M2 that are not embedded consists of an iconic sign, namely the drawing of a dagger. The dagger (that also appears on the initial splash page) can be seen as a metonymic icon for Macbeth. The dagger links therefore have an anchor that refers to the narrated story, to the main character of the play. Such an anchor is thematically homogenous with the narration, but it has no semantic relationship to the target node content. Therefore, here, at least the first time the reader interprets the link, the incertitude about the content of the target node is bigger and it also affects the kind of content (the anchor does not allow one to infer that the information provided by the target node will be an explanation). Correspondingly, the range of possible implicatures is wider. It is so wide that the reader cannot identify and formulate it. Therefore, at the end, it is reduced to the promise of relevance. Despite the iconic character of the anchor itself, at least the first time the reader interprets the link, such links are interpreted through a symptomatic process of interpretation: the reader trusts the promise of relevance the simple presence of the link manifests and recognizes the existence of a causal relationship between the link and the target node. Later on, during the navigation, the process 

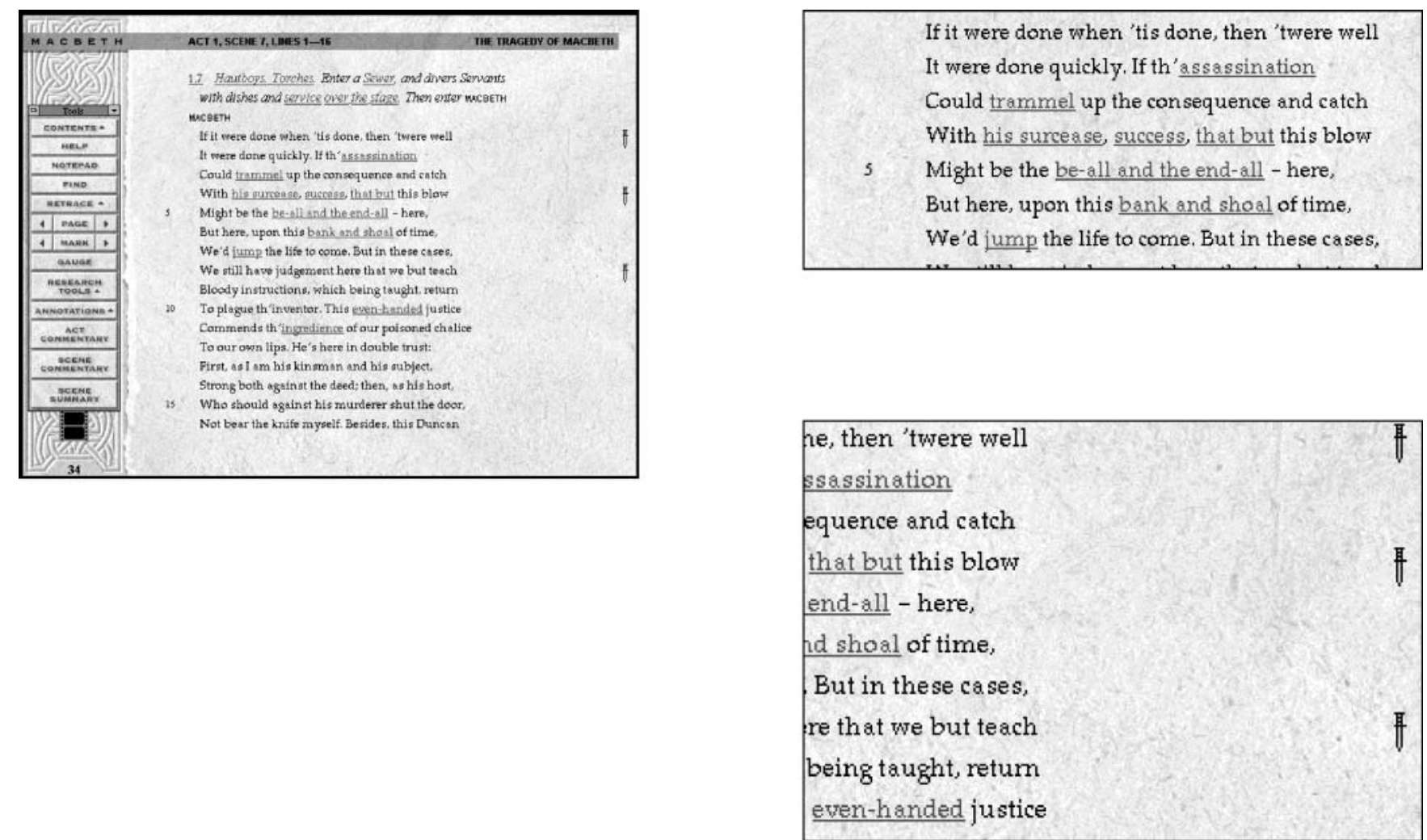
of interpretation becomes symbolic. In the perspective of a dialogue, the dagger link corresponds to an author's utterance of the kind 'This hyperlink counts as relevant information of an undefined kind of the passage of the text side to which the link is set and the boundaries of which will be clear after having read the information itself.'

Also, in the text pages of M3, two kinds of link - one embedded and one not - provide access to annotations (cf. figure 10). The textual embedded links provide access to a strictly linguistic explanation (synonyms, paraphrase, or translation into modern English). The links that are not embedded provide access to explanations of thematic, intratextual comments, reminders to elements of the story. The anchor of these links consists of an icon displaying a magnifying lens and the word 'Byte.' The magnifying lens focuses the reader on the aspect of closer and in-depth inspection. In this way, the idea that the target node contains more in depth explanations with respect to the literal comprehension of the text plays a role in first level inference. It contributes to restrict the range of possible implicatures to implicatures of the type 'This passage will be analyzed in greater depth.' These links are similar to the question mark links of M1. They are interpreted through an iconic process of interpretation, in which an association is expected to exist between the passage of the text to which the link refers and the kind of content of the target node (which is expected to be an explanation of aspects of a not very well delimited portion of text going beyond the clarification of the literal meaning of the words). In the perspective of a dialogue, the 'Byte' link (and its target node) corresponds to an author's utterance of the kind 'This hyperlink counts as a relevant explanation of the passage of the text side to which the link is set and the boundaries of which will be clear after having read the explanation itself; the scope of the explanation is constituted by deep aspects of the text passage, remaining beyond the literal meaning.'

In Lasting Image, the role played by the anchor in the generation of different dialogues is even more evident. The relationship between anchor and kinds of dialogue and utterance is very different for the two kinds of available links. The arrow hyperlinks available at the bottom of each page suggest an association between the arrow direction (backward/ forward) and the content of the target node (previous/following part of the story according to the logical order). The meaning relationship existing between source and target node is very explicit. The shape of the anchor does not give rise to a wide range of possible implicatures. The correspondence between first and second level of inference is clear and guaranteed. Because of their visibility and because of their arrow shape, hyperlinks of this type correspond to constitutive rules of the kind 'This hyperlink counts as a statement of their best suitability and as an 

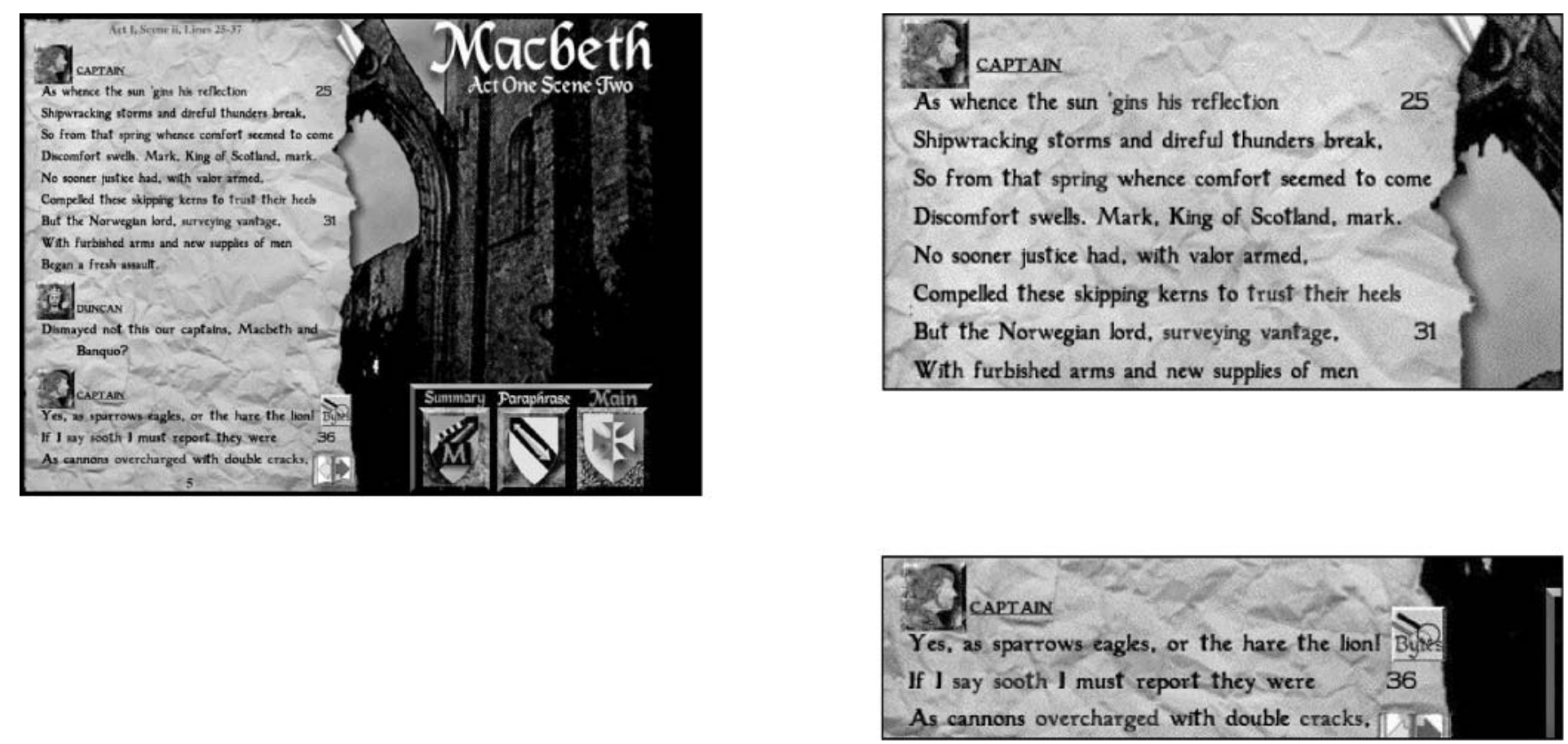

Figure 10. In M3, 'Bytes' and textual embedded hyperlinks are available on each text page 
assurance of the existence of a strict semantic relationship between the source and the target node.' They correspond to author's utterances in which the author narrates and guarantees that his narration proceeds in a logical and coherent way (cf. figure 11).

On the contrary, the invisible hyperlinks give rise to a wide range of implicatures. First of all, their character of invisibility adds to the interpretation (and therefore also to the dialogue) of an element of mystery and surprise. This character suggests to the reader that everything is possible. At the same time, at least at the beginning of his navigation, the reader supposes the existence of an association between the part of the image or the meaning of the words composing the anchor and the target node. Sometimes, this association actually exists. It is the case of the hyperlink signalled by the words 'blind man' of the text of the node 'Blind Man,' bringing the reader on the node 'Red Pagoda,' where the identity of the just introduced main character is specified (cf. figure 12).

It is also the case of the links 'photographs' on the nodes 'Bay Hills' and 'Sampans,' both bringing the reader on the node 'Full Deck,' in which a series of superposed photographs is displayed (cf. figure 13).

Finally, the link 'red bridge' on the node 'Fuji' leads the reader to the node 'redbridgerose' (which presents an image of the red bridge with a poetic description of it) and the link corresponding to the part of the image representing the shore leads to the node 'Ocean' (which presents an image suggesting an underwater view of a water expanse; cf. figure 14).

However, during the navigation, the reader realizes that most of the time such an association exists only between the whole content of the source node and the content of the target node. This happens, for example, in the links corresponding to parts of the image of the entry page (in which the habit of the narrator and his companions to go to a small village near Yokohama for drinking beer is described) and in the link 'pinkish pearl flesh' on the same page. All of these links lead to the node 'Blind Man,' in which the main character is introduced ('There was a blind man in the village ...'; cf. figure 15).

In realizing that the relationship between source and target node is of this nature, the reader understands that most of the time the anchor does not have a direct relationship with the content of the target node. In other words, the anchor does not provide useful hints for first level inference. Besides, unlike what happens with the arrow links, the reader cannot rely on the certitude that a logical reading order will be maintained. For this reason, the first level inference is reduced to the promise of relevance and, on such a basis, the second level inference will consist in looking for any possible and logical relationship that justifies the connection between source and target node. There also are some (rare) cases in which the 

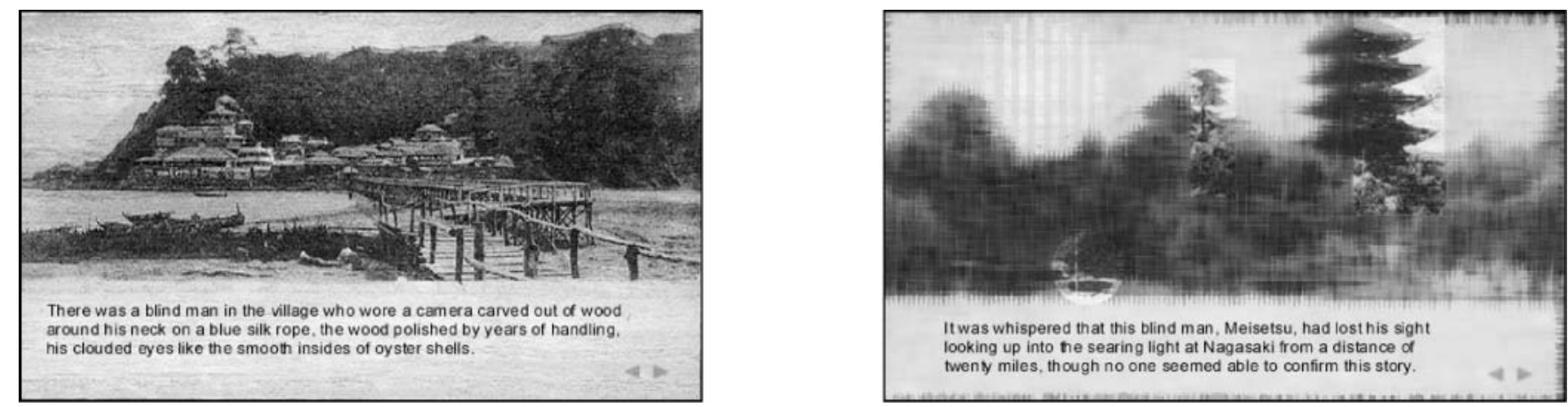

Figure 11. In Lasting Image, the forward arrow hyperlink available on the page 'Blind Man' (left) leads to a page 'Red Pagoda' (right), in which the cause of the blindness of the main character is explained 

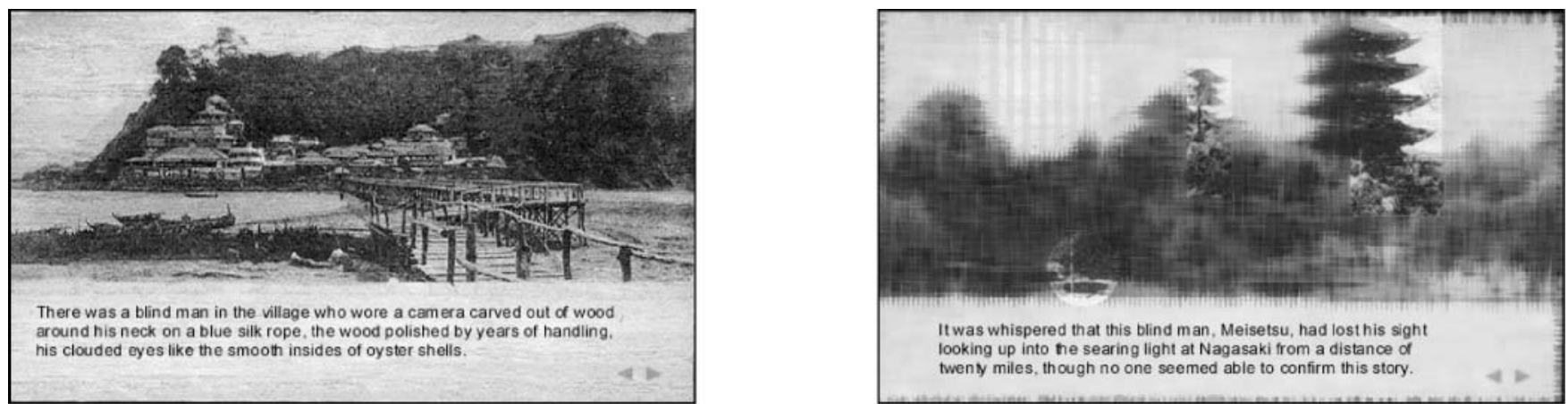

i

Figure 12. In Lasting Image, the words 'blind man' contained in the text of the node 'Blind man' (left) constitute the anchor of a hyperlink leading on the page 'Red Pagoda' (right), in which the cause of the blindness of the main character is explained 

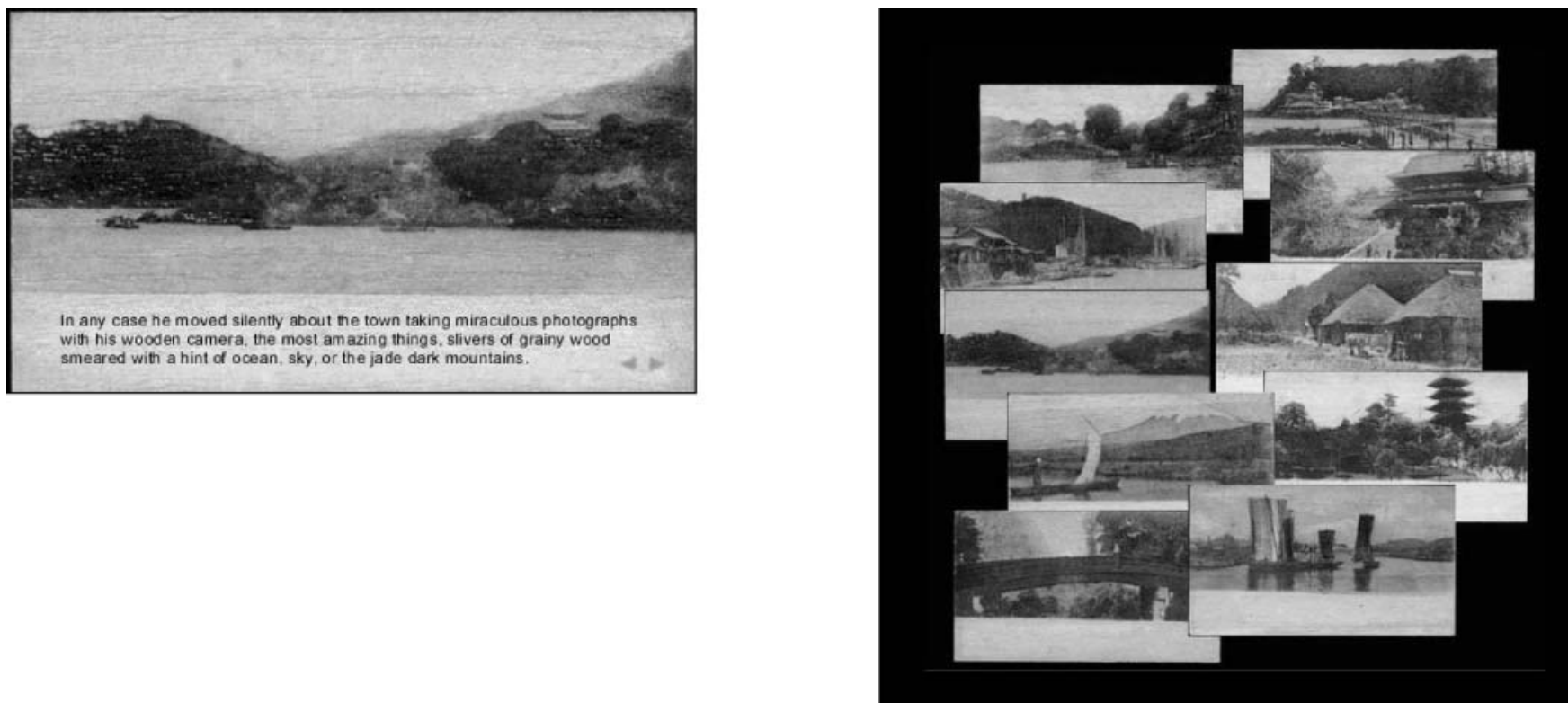

Figure 13. In Lasting Image, the word 'photographs' contained in the text of the node 'Bay Hills' (left) constitutes the anchor of a hyperlink leading on the page 'Full Deck' (right), displaying a series of superposed photographs 

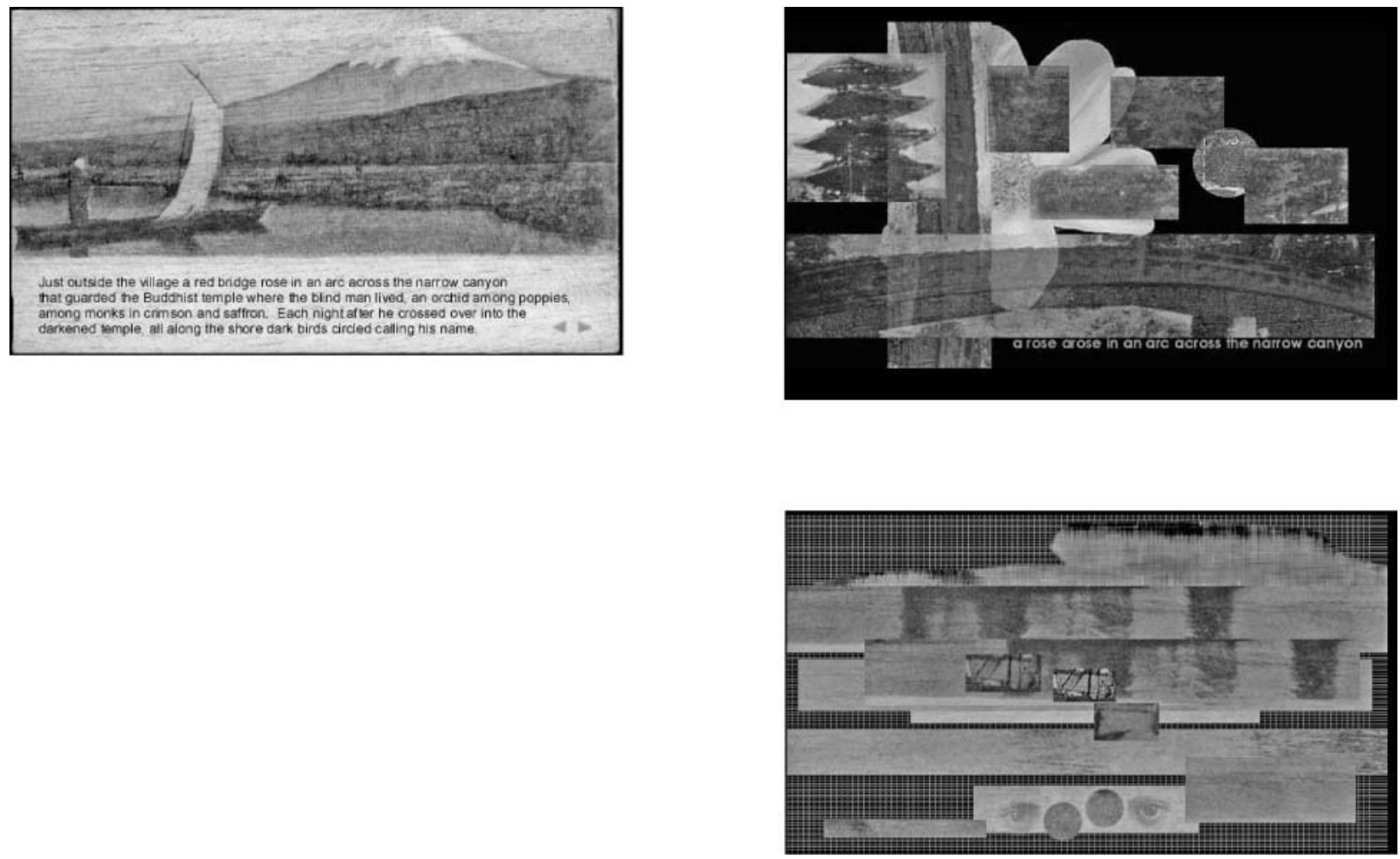

Figure 14. In Lasting Image, the words 'red bridge' contained in the text of the node 'Fuji' (left) constitute the anchor of a hyperlink leading on the page 'Redbridgerose' (top right), presenting an image of the red bridge and a poetic description of it. Correspondingly, the shore on the image of the node 'Fuji' is a hyperlink leading to an underwater view of a water expanse (the node 'Ocean,' bottom right) 

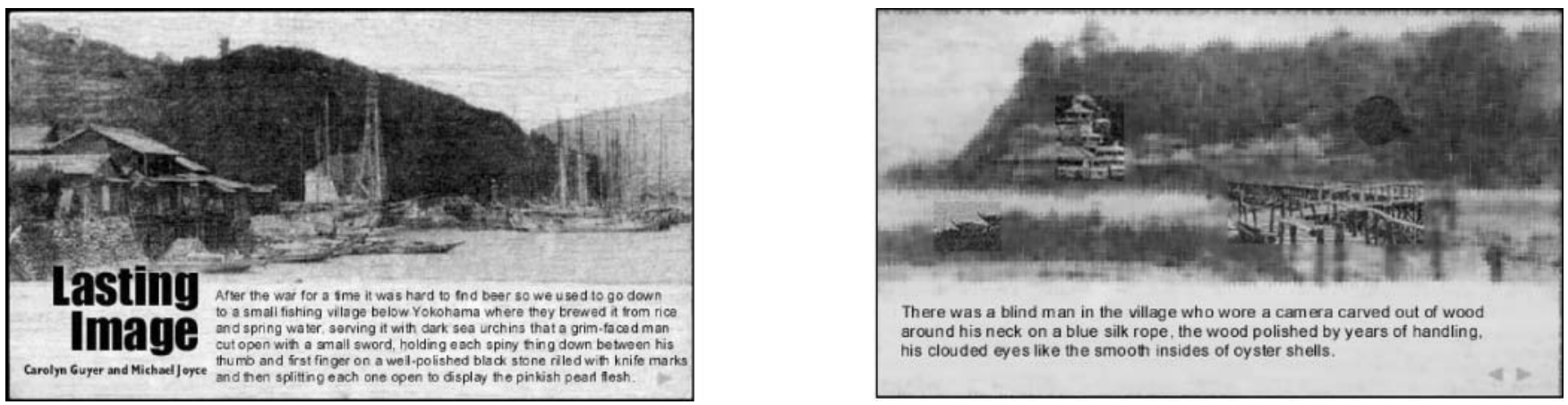

Figure 15. In Lasting Image, various links on the entry page (shown left, the anchor of which corresponds to parts of the image and to the words 'pinkish pearl flesh' in the text) lead to the page 'Blind Man' (right), in which the main character is introduced 
association does not exist. In such cases, the game of disorientation clearly appears in order to push the reader to continue the navigation in trying to construct a clearer meaning thanks to the contribution of other nodes. This is the case with the 'spiny thing' links on the entry page (where the initial situation is described) and the case of the 'whispered' link on the node 'Red Pagoda' (where the past of the main character is narrated). Both of these links lead to the node 'Distant Adobes,' in which the narrator describes the card game the soldiers play with the photographs taken by the main character (cf. figure 16).

All of the examples we described show that the anchor plays an important role in defining the kind of dialogue and utterances corresponding to the hyperlinks. All our observations emerging from the consideration of different cases can be resumed in a central problem. Namely, the problem is to know if an association exists only between the source and the target nodes or if an association exists also between the anchor and the target node. In the first case, the anchor means almost nothing, because it does not provide useful hints for the first level inference. What counts in these cases is the promise of relevance. In the second case, the anchor provides hints capable of giving rise to a more or less wide range of implicatures that will be confirmed or reversed by the association with the content of the target node at the second level inference.

\section{Conclusions}

In the study of hypertextual communication, attention is usually paid to the importance of the role played by the reader/user and on his interaction with the application. At the very beginning of hypertext studies, a perspective that argued for the death of the author was emphasized (cf. for instance Bolter 1991; Delany and Landow 1994; Joyce 1995; Landow 1997, who refers to Foucault's and Barthes' essays on this topic). Since then, the practice of hypermedia design and the studies on hypermedia consumption, web usability, and requirement analysis have been proving that such an argument cannot be maintained, by showing that the choices made in designing and implementing the application deeply condition the user's navigation freedom. The crucial role played by the author is therefore acknowledged (cf., for example, Cantoni and Paolini 2001). However, it is generally maintained that navigating in a hypertextual application constitutes a dialogue between the user and the application itself. The interaction of a user with a hypertext is a dialogue in which the application proposes possible choices and asks the user which one of these choices he prefers and the reader answers in expressing his choice among 

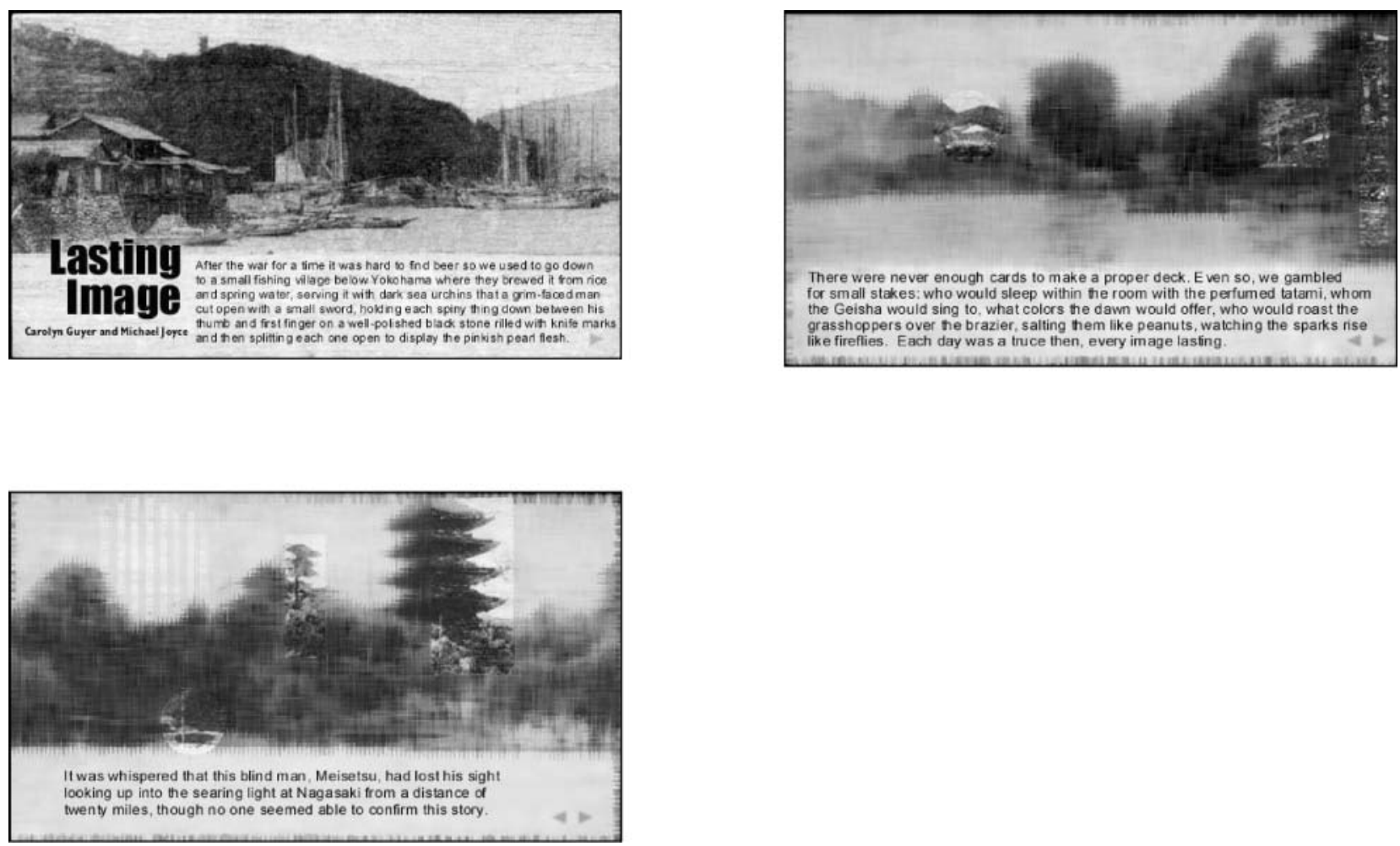

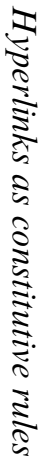

Figure 16. In Lasting Image, the words 'spiny thing' (contained in the text of the entry page, shown top left) and 'whispered' (contained in the text of the node 'Red Pagoda,' shown bottom left) constitute the anchor of hyperlinks leading to the page 'Distant Adobes' (right) 
the proposed possibilities (cf., for example, Pajares Tosca 2000: 79; Cantoni and Paolini 2001: 43; Di Blas and Paolini 2003). In this common perspective, the process starting from the hypertext production and ending at the hypertext consumption is seen as constituting two separated phases.

Such a perspective relies on some unquestionable facts. First, hypertextual communication, as all other kinds of written communication, is asynchronous. Therefore, the situation and context of text production are different and distinct with respect to all the possible situations and contexts of reception. Second, because of text fragmentation and multilinearity, in respect to other kinds of written texts, hypertext requires of the reader greater and more complex interpretation work.

The characterization of hyperlinks within the constitutive rule of hypertextual communication underlines the essential role hyperlinks play in the creation of different dialogues. These dialogues do not only depend on the reader/user's choices. They depend in a decisive manner on the author/ designer's choices. The author/designer sets the constitutive rules of the hypertextual communication. Through these choices, he remains present in the hypertext and he aims at managing the possible misunderstandings that can arise due to the asynchronous character of this kind of communication. Every hypertext has an author who wants to communicate something and who makes given choices instead of others in order to succeed in this communication. To a certain extent, the reader can elaborate interpretations that were not foreseen by the author. However, through his choices, the author tries to carry the user to the recognition of his communicative intention and to the realization of goals that he considers to be common to himself and to the user. Particularly, by defining hyperlinks and their strategy of manifestation - that is, by controlling the performative nature of hyperlinks (cf. Cantoni and Paolini 2001: 42) and by controlling the appeal they exert on the user - the author aims at leading the hypertextual communication. By choosing to make available certain hyperlinks instead of others and by choosing given technical devices in order to manifest those hyperlinks, the author 'speaks' given utterances instead of others and he tries to control the reader activity and to direct the reader on the aspects that, in his view, are the most important ones. The author remains present in hypertext: his choices reveal him. Instead of 'killing' the author, hypertext strengthens his role as designer of paths of meaning.

\section{Notes}

1. We refer to the concept and classification of processes of sign interpretation elaborated by Peter Schulz (2000). 
2. The nature of the sign composing the anchor and the nature of the interpretation process of the link can be different. This happens, for example, with embedded verbal hyperlinks. Their anchor is composed of a symbolic sign (words), but they are interpreted through an iconic process of interpretation since it is based on the recognition of an associative relationship existing between the meaning of the words composing the anchor and the content of the target node.

3. From a technological standpoint, this statement is imprecise. Hypermedial applications are automatically generated starting from databases. Therefore, properly speaking, the author of the application does not establish the hyperlinks, but the rules the software will employ for their automatic generation (cf. Cantoni and Paolini 2001: 40, 45). Hyperlinks are the result of the combination of these rules. However, at the communicative level (at the level of the relationship author-application-reader in which the author aims at communicating to the reader a given meaning), we focus our attention on the 'final product,' that is, on hyperlinks themselves, and not on the rules lying at their generation.

\section{References}

BBC Education (1995). Macbeth by William Shakespeare. CD-ROM. London: Harper Collins.

-(1996). A Midsummer Night's Dream by William Shakespeare. CD-ROM. London: Harper Collins.

Bolter, Jay David (1991). Writing Space: The Computer, Hypertexts, and the History of Writing. Hillsdale, NJ: Lawrence Erlbaum.

-(2001). Writing Space: Computers, Hypertext, and the Remediation of Print. Hillsdale, NJ: Lawrence Erlbaum.

Bolter, Jay David and Grusin, Richard (1999). Remediation: Understanding New Media. Cambridge, MA: MIT Press.

Bride Digital Classics (1999). Macbeth by William Shakespeare. CD-ROM. Dedham, MA: Bride Media International.

Bühler, Karl (1999 [1934]). Sprachtheorie. Die Darstellungsfunktion der Sprache. Stuttgart: Lucius and Lucius Verlagsgesellschaft.

Cantoni, Lorenzo and Paolini, Paolo (2001). Hypermedia analysis. Some insights from semiotics and ancient rhetoric. Studies in Communication Sciences/Studi di Scienze della comunicazione 1, 33-53.

Conklin, Jeff (1987). Hypertext: An introduction and survey. IEEE Computer 20 (9), $17-$ 41.

Delany, Paul and Landow, George P. (1994). Hypertext, hypermedia, and literary studies: The state of the art. In Hypermedia and Literary Studies, Paul Delany and George P. Landow (eds.), 3-50. Cambridge, MA: MIT Press.

Di Blas, Nicoletta and Paolini, Paolo (2003). There and back again. What happens to phoric elements in a 'web dialogue.' Document Design 4 (3), 194-206.

Douglas, J. Yellowlees and Hargadon, Andrew (2000). The pleasure principle: Immersion, engagement, and flow. In Hypertext '00: Proceedings of the Eleventh ACM Conference on Hypertext and Hypermedia, Frank M. Shipman et al. (eds.), 153-160. New York: Association for Computing Machinery.

Fagerjord, Anders (2001). Issues of sequence in converging media: Studying world wide web documentaries. In COSIGN2001. Proceedings of the 1st Conference on Computational 
Semiotics for Games and New Media. Available online at http://www.kinonet.com/ conferences/cosign2001/pdfs/cosign2001.pdf.

Grice, H. Paul (1995 [1975]). Logica e conversazione. In Gli atti linguistici. Aspetti e problemi di filosofia del linguaggio, Marina Sbisà (ed.), 199-219. Bologna: Feltrinelli.

Guyer, Carolyn and Joyce, Michael (2000). Lasting Image. Available online at http:// www.eastgate.com/LastingImage/Welcome.html.

Joyce, Michael (1995). Of Two Minds: Hypertext Pedagogy and Poetics. Ann Arbor: University of Michigan Press.

Landow, George P. (1997). Hypertext 2.0. The Convergence of Contemporary Critical Theory and Technology. Baltimore: John Hopkins University Press.

lingo.uib (eds.) (1999). A Midsummer Night's Dream by William Shakespeare. Bergen: LINGO, University of Bergen. Available online at http://cmc.uib.no/dream/index.html.

Nelson, Theodor H. (1990). Literary Machines. Mindful.

Pajares Tosca, Susan (1999). The lyrical quality of links. In Hypertext '99: Returning to Our Diverse Roots: The 10th ACM Conference on Hypertext and Hypermedia, Klaus Tochtermann et al. (eds.), 217-218. New York: Association for Computing Machinery.

- (2000). A pragmatics of links. In Hypertext '00: Proceedings of the Eleventh ACM Conference on Hypertext and Hypermedia, Frank M. Shipman et al. (eds.), 77-84. New York: Association for Computing Machinery.

Pilkington, Adrian (2000). Poetic Effects: A Relevance Theory Perspective. Amsterdam: John Benjamins.

Searle, John R. (1995 [1969]). Speech Acts: An Essay in the Philosophy of Language. Cambridge: Cambridge University Press.

Schulz, Peter (2000). The risk of communication. A semiotic approach. Paper presented at the Conference of the Semiotic Society of America, Toronto.

Slatin, John M. (1990). Reading hypertext: Order and coherence in a new medium. College English 52 (8), 870-883.

Voyager (1994). Macbeth by William Shakespeare. CD-ROM. New York: Voyager.

Wenz, Karin (1997). Principles of spatialization in text and hypertext. In Semiotics of the Media. State of the Art, Projects, and Perspectives, Winfried Nöth (ed.), 575-586. Berlin/ New York: Mouton de Gruyter.

Wingert, Bernd (1999). Der Leser im Hypertext — Im Weinberg oder im Steinbruch? In Hyperfiction. Hyperliterarisches Lesebuch: Internet und Literatur, B. Suter and M. Böhler (eds), 159-172. Basel: Stroemfeld. Available online at http://www.cyberfiction.ch/beluga/ digital/99/wingert.htm.

Wirth, Uwe (2002). As we may surf: The relevance of abductive inference for surfing through the internet. Semiotica 141 (1/4), 159-168.

Sabrina Mazzali-Lurati (b. 1971) is an Assistant Lecture in the Faculty of Communication Sciences at the University of Lugano〈sabrina.mazzali-lurati@lu.unisi.ch〉. Her research interests include literature and hypermedia, semiotics of new media, and composition. Her publications include 'The actualization of reading strategies in hypermedia' (with P. Shulz, 2003); 'A semiotic approach to hypermedia for literary studies. New conditions for the act of reading? (with P. Shulz, 2004); 'Photography: Semiotics' (with L. Cantoni, 2006). 